Revue d'histoire de l'enfance « irrégulière »

Le Temps de l'histoire

16 | 2014

La part scolaire : jeunesse irrégulière et école $\left(\mathrm{XIX}^{\mathrm{e}}\right.$ $\mathrm{XX}$ siècles)

\title{
Introduction au dossier : « La part scolaire »
}

\section{Laurent Besse}

\section{(2) OpenEdition \\ Journals}

Édition électronique

URL : https://journals.openedition.org/rhei/3586

DOI : $10.4000 /$ rhei.3586

ISBN : 978-2-7535-4019-4

ISSN : $1777-540 \mathrm{X}$

Éditeur

Presses universitaires de Rennes

Édition imprimée

Date de publication : 30 octobre 2014

Pagination : 23-29

ISBN : 978-2-7535-3558-9

ISSN : $1287-2431$

\section{Référence électronique}

Laurent Besse, «Introduction au dossier : «La part scolaire » », Revue d'histoire de l'enfance «irrégulière » [En ligne], 16 | 2014, mis en ligne le 30 octobre 2014, consulté le 09 septembre 2021. URL : http:// journals.openedition.org/rhei/3586; DOI : https://doi.org/10.4000/rhei.3586

Ce document a été généré automatiquement le 9 septembre 2021.

(c) PUR 


\title{
Introduction au dossier : « La part scolaire »
}

\author{
Laurent Besse
}

1 La jeunesse contemporaine est le produit de l'école: le développement de la scolarisation, primaire au $\mathrm{XIX}^{\mathrm{e}}$ siècle puis secondaire au $\mathrm{xx}^{\mathrm{e}}$ siècle, a contribué à faire de l'élève la figure normale de l'enfance puis de l'adolescence, au point de rendre parfois difficile à concevoir qu'il puisse exister des enfants échappant à l'emprise scolaire. Sous cet aspect, la jeunesse irrégulière mérite donc particulièrement son nom: elle est étrangère à ce qui devient progressivement la règle pour la majorité des jeunes et n'a droit qu'à une scolarisation limitée, tant dans ses ambitions que dans sa régularité ou sa durée. Ainsi, malgré l'existence ancienne d'instituteurs de la Pénitentiaire, la part dévolue à l'étude dans les institutions de prise en charge des mineurs de justice a toujours été réduite, même lorsqu'à partir de la fin du XIX ${ }^{e}$ siècle, les préoccupations de réhabilitation des jeunes délinquants émergent progressivement au côté de la volonté répressive traditionnelle. Cela n'est pas le moindre des paradoxes : voilà des jeunes qui, par définition, étaient aux mains mêmes de l'État, mais qui ne recevaient que des miettes d'instruction ${ }^{1}$. Si le projet de l'école primaire républicaine de Jules Ferry a pu être présenté de manière schématique comme une éducation par l'instruction, ce qui était offert à la jeunesse délinquante produisait dans les faits une éducationrééducation sans instruction, loin de l'universalité proclamée du droit à l'instruction. La situation des pupilles de l'Assistance publique était meilleure que celle des enfants de justice, du moins jusqu'à la première guerre mondiale, puisque l'Assistance s'efforça de «relever le défi des lois Ferry ${ }^{2}$ ». Mais cet effort n'alla pas au-delà de l'alphabétisation minimum et ne déboucha pas après 1918 sur un projet de scolarisation plus ambitieuse.

2 La généralisation de l'instruction puis son obligation à partir de 1882, imposaient de nouvelles normes, celle de l'assiduité scolaire, celle du bon élève, qui produisaient leur lot de déviances : enfants fugueurs fuyant l'école, familles paysannes pauvres faisant un usage stratégique de la fréquentation scolaire jusque dans les années 1930, violences 
scolaires, etc. Dans la fabrique sociale de la délinquance, l'école joue donc également son rôle.

3 L'obligation d'instruction pose également la question de la scolarisation des enfants différents, « inadaptés ", « instables », « handicapés », «à besoins spécifiques », quels que soient les noms qu'on leur donne. L'article 4 de la loi de 1882 évoque les sourdsmuets et aveugles dont il convient de réaliser l'instruction primaire, mais passe sous silence les autres. Pour ce qui concerne les "arriérés», on connaît l'importance symbolique de la loi du 15 avril 1909 qui ne commencera à donner des effets qu'à partir du Front populaire, faute en particulier d'une formation des maîtres ${ }^{3}$.

4 Les différentes catégories d'enfants qui viennent d'être évoquées ont en commun leur éloignement de l'école ordinaire, «l'école régulière » selon l'expression utilisée par les Québécois ${ }^{4}$. Mais elles relèvent d'institutions spécialisées qui ont généré des champs de recherche eux-mêmes spécifiques, qui ont naturellement conduit à segmenter les recherches, dont celles portant sur les questions scolaires ${ }^{5}$. Seule la catégorie de l'inadaptation, portée par les milieux médicaux et judiciaires a pu, au milieu du $\mathrm{xx}^{\mathrm{e}}$ siècle, prétendre rassembler ces populations dans un ensemble unifiant. Mais notons que ce phénomène est contemporain d'un effacement relatif de l'Éducation nationale sur ces questions ${ }^{6}$. Selon les champs institutionnels et donc les champs de recherche, l'histoire de l'instruction a fait l'objet d'investissements très différents. Si l'éducation spéciale a donné lieu à des travaux spécifiques ${ }^{7}$, l'histoire de l'éducation spécialisée a produit beaucoup moins de recherches consacrées à la "part scolaire ", pas même pour en relever les lacunes ou la faiblesse. En forçant un peu le trait, on pourrait écrire que ce silence (relatif) de la recherche a reproduit l'opposition entre l'éducateur et l'instituteur, si forte dans ce milieu ${ }^{8}$. Quant à l'histoire de l'école, parfois assimilée au tout de l'éducation par ses tenants, elle s'est souciée d'abord du cœur du système éducatif et n'a accordé qu'une attention marginale aux marges du système. De plus, elle n'a abordé qu'assez récemment la scolarisation des milieux populaires urbains, ce qui ne facilitait pas nécessairement la rencontre avec les mineurs de justice ${ }^{9}$.

5 L'appel à contribution ${ }^{10}$ dont découle ce dossier visait à découvrir des recherches récentes sur l'instruction, là où elle ne va pas de soi, là où la dimension éducative (ou réputée telle) prime, dessinant un envers de l'école ordinaire ou "régulière » où l'exposition aux savoirs est considérée comme prioritaire. Les articles réunis concernent la France, mais avec deux éclairages étrangers sur l'Italie et le Québec. Quant à la période chronologique couverte, elle s'étend sur un siècle, de la généralisation de la scolarité obligatoire aux années 1980. Par ailleurs, les articles témoignent de la diversité des champs investis par la recherche, puisque deux concernent l'enseignement spécial (ou adapté), l'un l'éducation spécialisée et enfin, deux l'institution scolaire dans sa relation à des populations qu'elle tient pour étrangères, les enfants de la rue et les Tsiganes. Ces deux dernières contributions interrogent les normes scolaires ordinaires et la capacité de l'institution et des populations à pratiquer des accommodements avec elles.

\section{L'école face à ceux qui lui échappent}

6 Les articles de Delphine Pietu et d'Alice Sarcinelli se complètent par leur angle d'attaque opposé : une monographie d'une association parisienne à la toute fin du $\mathrm{xIX}^{\mathrm{e}}$ siècle, sur un temps court, auquel répond une mise en perspective de plus d'un siècle de 
scolarisation des Tsiganes en Italie, replacée dans un contexte européen. Delphine Pietu, jeune historienne, retrace l'histoire d'un échec, celui de la Société contre la mendicité des enfants qui s'efforçait de scolariser les enfants pour concurrencer l'école de la rue. Cette action se heurte aux limites de l'institution scolaire, dont la résistance de certains maîtres à admettre des enfants qui perturbent l'ordre de la classe. Mais ce sont également les enfants et leur famille qui s'opposent par un absentéisme perlé ou la pratique de la mendicité en dehors des heures de classe, ce qui oblige la Société à admettre qu'elle n'a fait que déplacer le problème et que l'institution scolaire n'a pas tous les pouvoirs qu'on voudrait lui prêter en cette fin de XIX ${ }^{e}$ siècle $^{11}$.

7 Les premiers efforts italiens pour scolariser les Tsiganes sont contemporains car, comme le rappelle Alice Sarcinelli, il existe un lien fort entre unification nationale et tentatives pour «nationaliser» en les scolarisant les populations perçues comme allogènes ${ }^{12}$. D'emblée, les élèves tsiganes ont été rangés dans la catégorie des arriérés et relèvent de l'enseignement spécial. Leur prise en charge s'inscrira dans une logique d'assimilation mais par la mise en place d'une scolarité différentielle promue par des milieux qui se veulent leurs défenseurs. Alice Sarcinelli donne très bien à voir la construction d'une catégorie compatible avec la logique scolaire, « l'élève tsigane » qui devient de fait "l'enfant tsigane ». Cette catégorie est construite sur fond d'expertises psychologiques utilisées de manière pour le moins hasardeuse. Surtout l'auteure donne un très bon exemple de la perméabilité des catégories qu'on assigne aux jeunes irréguliers, puisque les jeunes tsiganes sont un temps assimilés à des arriérés avant de l'être aux jeunes migrants - alors qu'ils sont tous italiens - sous l'effet des réflexions européennes sur le droit des minorités au tournant des années 1990.

\section{Éducation nationale et monde associatif}

Deux contributions abordent l'engagement associatif d'inspecteurs pour faire avancer la cause de l'éducation spéciale, mais dans des contextes fort différents. L'article de Brigitte Hébert Vassogne apporte une richesse d'informations inédites sur le rôle joué par des inspecteurs de l'Instruction publique dans l'entre-deux-guerres. Ces fonctionnaires cherchent à compenser les lacunes de leur institution par un engagement militant. Trois figures individuelles (un homme, deux femmes) sont mises en avant mais c'est tout un monde qui est finement restitué dans son rôle de passeur entre l'univers médical, les associations philanthropiques, les instituteurs et l'Éducation nouvelle. On découvre des lieux (les jurys de prix et concours), des bulletins dont on connaît le rôle dans la formation des instituteurs, mais également des cours de préparation à l'examen du CAEA (certificat d'aptitude à l'enseignement des arriérés) que des associations regroupées organisent, faute d'initiative officielle en ce domaine. Les réseaux étudiés sont parisiens, il importe de le souligner, mais ils ont des liens avec la province. Au moment du Front populaire, ces inspecteurs jouent un rôle important auprès des autorités qui ont la volonté de rompre avec l'inertie qui prévalait jusqu'alors. Un précieux répertoire de fonctionnaires de l'Instruction publique repérés pour leur action administrative et militante complète l'article.

9 André Catteaux est le collègue de ces inspecteurs, mais il appartient à la génération suivante, celle qui dispose des moyens pour construire l'enseignement spécialisé dans l'après-guerre. Il livre un témoignage complémentaire, portant sur les liens entre l'administration de l'Éducation nationale et sa périphérie d'œuvres laïques, si 
importante dans le domaine de l'éducation populaire et de l'enfance inadaptée. L'interpénétration entre Éducation nationale et secteur "privé » repose sur les présidences de droit et de fait assurées par les inspecteurs, sur la multi-position des militants de la sphère laïque (on retrouve les mêmes militants dans différentes associations) ainsi que sur les MAD (mis-à-disposition), ces enseignants rémunérés par l'Éducation nationale mais effectuant leur service dans une œuvre. Ces phénomènes sont souvent mal connus et sont ici décrits à hauteur d'homme.

\section{L'instruction comme condition de l'éducation chez les jeunes délinquants}

10 Ce programme - à faire rêver le plus républicain des défenseurs de l'école de Jules Ferry - c'est celui que nous décrivent Louise Bienvenue et Andréanne LeBrun, à propos de la célèbre institution de Boscoville au Québec. Au centre de leur étude, non pas l'institution éducative mais une méthode, « le Boulot » qui vise à permettre aux jeunes, appelés "citoyens", de s'instruire à l'intérieur de l'institution et à s'approprier des savoirs équivalents à ceux acquis par les élèves du système scolaire ordinaire ou « régulier ». Le "Boulot» est en une méthode de travail par fiches, élaborée à Boscoville, permettant une individualisation poussée, inspirée par l'Éducation nouvelle. L'instruction générale est conçue comme une manière de redonner confiance aux jeunes, ce qui contraste avec la priorité donnée aux apprentissages professionnels dans les autres institutions tant au Québec qu'en France. On notera également que dans cet univers très masculin par son public de jeunes garçons et son personnel, ce sont des femmes qui ont la charge du travail d'élaboration des fiches, sans qu'on sache très bien ce qu'il faut en conclure quant au statut de cette part du travail éducatif...

11 Ajoutons une dernière raison de nous réjouir de cet article: l'une des critiques récurrentes adressées à l'histoire de l'éducation porte sur sa tendance supposée à consacrer son énergie à retracer les conflits entre acteurs administratifs au lieu d'étudier la relation éducative entre éducateurs et éduqués qui seraient les grands oubliés de la recherche. Outre que ce préjugé est sans doute largement dépassé ${ }^{13}$, il attribue à l'histoire de l'éducation un travers commun à bien d'autres domaines. Pour ne citer qu'un exemple intéressant directement notre revue, l'histoire de la relation de soin en est encore au stade du défrichement en $2014^{14}$. Raison de plus malgré tout, de relever l'attention que les auteures portent à l'usage et à la perception que les jeunes avaient du "Boulot». Non seulement, elles scrutent les archives à la recherche des mots qui furent les leurs dans les années 1950 ou 1960, mais elles sont également allées à leur rencontre, pour essayer d'évaluer l'empreinte qu'a pu laisser cette expérience cinquante ans plus tard.

12 En guise, ni de regret, ni de remords, mais au contraire pour susciter des vocations, relevons deux domaines qui n'ont pas suscité de propositions d'articles, en dépit de l'intérêt qu'ils présentent. L'absence du premier domaine peut s'expliquer par sa proximité chronologique puisqu'il concerne la prise en charge des enfants handicapés par l'Éducation nationale après la loi en faveur des personnes handicapées du 30 juin 1975. Celle-ci n'a pas encore donné lieu à des recherches historiques, malgré l'existence de travaux sociologiques qui s'efforcent de replacer leur objet dans une perspective diachronique ${ }^{15}$. Cette période, on le sait, s'accompagne de la remise en cause de l'enseignement spécial et de la montée des demandes d'inclusion scolaire qui sont 
consacrées par la loi du 11 février 2005. L'action des familles au niveau local semble jouer un effet important, tant pour appuyer un mouvement d'inclusion dans les classes ordinaires que pour réclamer des structures spécifiques pour des enfants jusque-là considérés comme inéducables ${ }^{16}$. Des monographies paraissent donc nécessaires, pour comprendre les évolutions de la période, au-delà de statistiques qui restent imparfaites ${ }^{17}$.

Par ailleurs, l'histoire de la formation professionnelle à destination des jeunes « irréguliers» n'a pas été traitée. Pourtant l'opposition et la concurrence «travail versus travail scolaire" a été au cœur des modes de prise en charge depuis le XIX siècle ${ }^{18}$. Une période mériterait une histoire spécifique, au moins pour les jeunes placés : celle où, à partir de 1945 et jusqu'à la fin des Trente Glorieuses, la préparation au CAP (certificat d'aptitude professionnelle) se généralise dans les centres relevant de l'Éducation surveillée et les institutions privées de prise en charge des mineurs. L'atelier avec ses moniteurs, ses éducateurs techniques, acquiert une importance centrale. L'évolution s'inscrit dans la logique de scolarisation des apprentissages professionnels qui se met alors en place en France, avec le CAP comme horizon pour les catégories populaires et la création des centres d'apprentissage, ancêtres des lycées professionnels ${ }^{19}$. Par ailleurs, l'enseignement professionnel porté, du moins jusqu'aux années 1960, par une volonté de former "l'homme, le travailleur et le citoyen », ancré dans une culture ouvrière, est un espace d'expérimentation pédagogique, en lien avec les mouvements d'éducation populaire ${ }^{20}$. Cette culture de l'enseignement technique était plus compatible avec les conceptions de l'éducation spécialisée que celle des enseignants de l'école ordinaire. Surtout pour la première fois sans doute, les jeunes en institution bénéficiaient d'une formation qui était en phase avec les évolutions globales de la scolarisation des autres jeunes. Il y a là un chantier intéressant.

\section{NOTES}

1. À propos de l'instruction des jeunes détenus : YVOREL Élise, Les Enfants de l'ombre. La vie quotidienne des jeunes détenus au $\mathrm{XX}^{e}$ s. en France métropolitaine, Rennes, PUR, 2008, p. $158-180$.

2. JABLONKA Ivan, Ni père ni mère. Histoire des enfants de l'Assistance publique (1874-1939), Paris, Le Seuil, 2006, p. 194.

3. VIAL Monique, Les enfants anormaux à l'école: aux origines de l'éducation spécialisée, 1882-1909, Paris, Armand Colin, 1990 et RocA Jacqueline, De la ségrégation à l'intégration: l'éducation des enfantsinadaptés de 1909 à 1975, Vanves, CTNERHI, 1992.

4. Voir dans ce dossier l'article de Louise Bienvenue et Andréanne LeBrun.

5. Pour un exemple, à l'inverse, d'histoire des "points de convergence » entre deux catégories : GARDET Mathias, Orphelins et mineurs délinquants (1889-1959). Deux catégories et deux champs de recherche pour une enfance placée, HDR, Paris 8, 2012, p. 39-184. 
6. CHAUVIÈRE Michel, Enfance inadaptée : l'héritage de Vichy. L'Efficace des années quarante, Paris, l'Harmattan, 2009 ( $1^{\text {re }}$ édition 1980).

7. Voir en particulier ceux des auteures citées note 3.

8. CHAUVIÈRE Michel, fablet Dominique, «L'instituteur et l'éducateur. D'une différenciation historique à une coopération difficile ", Revue française de pédagogie, 2001, n' 134, p. 71-85.

9. La thèse de THIVEND Marianne, L'école républicaine en ville: Lyon 1870-1914, Paris, Belin, 2006, est la première monographie consacrée à la scolarisation dans une grande ville.

10. [http://calenda.org/253947] (consulté le 20 août 2014).

11. L'histoire retracée est strictement contemporaine du lancement de la campagne pour "les lendemains de l'école », lancée par la Ligue de l'enseignement en 1894 qui vise à élargir à l'adolescence les vertus de l'encadrement postscolaire.

12. L'article rejoint ici des perspectives abordées dans la revue dans BOUSQUET MariePierre, «Êtres libres ou sauvages à civiliser ? L'éducation des jeunes Amérindiens dans les pensionnats indiens au Québec, des années 1950 à 1970 ", Revue d'histoire de l'enfance «irrégulière », n 14, 2012, p. 163-192.

13. Pour s'en tenir aux élèves "réguliers » et à un essai français de synthèse : GRÈzESRUEFF François, LEDUC Jean, Histoire des élèves en France. De l'Ancien Régime à nos jours, Paris, Armand Colin, 2007.

14. Voir l'argumentaire de la journée d'études : "Le soin, histoire d'une relation", Paris, 4 juin 2014. [http://chsp.sciences-po.fr/evenement/le-soin-histoire-dunerelation] consulté le 20 août 2014.

15. DUPONT Hugo, Ni fou ni gogol. Ségrégation scolaire et réaction aux stigmates des jeunes orientés en ITEP, thèse de sociologie, Bordeaux 2, 2013. L'auteur ne peut s'appuyer sur des travaux d'histoire que jusqu'aux années 1950 (p. 47-73).

16. LERCH Dominique, "Séparer, intégrer, inclure. Les enfants handicapés à l'école ", Ethnologie française, 3/2009, vol. 39, p. 447.

17. Ibid.

18. Comme le montrent également Louise Bienvenue et Andréanne LeBrun à propos du Québec.

19. Voir les travaux de TROGER Vincent, PELPEL Patrice, Histoire de l'enseignement technique, Paris, l'Harmattan, 2001 ( $1^{\text {re }}$ édition Hachette éducation 1993).

20. Les AROEVEN (Associations régionales des œuvres de vacances de l'Éducation nationale) sont nées dans l'enseignement technique (ex-AROEVET, ET pour enseignement technique). 


\section{AUTEUR}

\section{LAURENT BESSE}

Maître de conférences en histoire contemporaine, IUT de Tours, CeTHiS (EA 6298). 